\section{Digital clinical records and practice administration in primary dental care}

\author{
I.-V. Wagner, ${ }^{1}$ R. S. Ireland ${ }^{2}$ and K. A. Eaton ${ }^{3}$
}

IN BRIEF

- This paper describes in detail the development of digital clinical records and digital practice administration.

- It highlights the need for all digital equipment to be fully integrated into one system in each operatory.

- It considers how the 'digital revolution' has impacted upon practice administration.

Usually, a 'computerised dental practice' has included a series of diagnostic instruments, intra-oral cameras, digital radiographic systems, treatment planning systems, CAD-CAM systems, management systems etc. However, these 'island solutions' have not been integrated into one system. Nevertheless, it is possible to produce fully integrated systems for digital clinical records, based on established physiologic and cognitive-ergonomic concepts. The first part of this paper outlines the philosophy behind the development of such a totally integrated system for digital clinical records. The second - digital practice administration - considers how the 'digital revolution' has impacted upon practice administration.

\section{E-LEARNING IN DENTISTRY}

Section A: Teaching and technology

1. A description of the new technologies used in transforming dental education

2. Seeing is believing: dental education benefits from developments in videoconferencing

3. Webcasting: casting the web more widely

4. Top of the pops - CD-ROM and DVDs in dental education

Section B: Informatics: better informed by systems and services

5. Better informed: an overview of health informatics

6. Better informed in clinical practice a brief overview of dental informatics

7. Digital clinical records and practice administration in primary dental care

Section C: Impact of e-learning in

dental education

8. Remember the days in the old school yard: from lectures to online learning

9. An intricate web - designing and authoring a web-based course

10. The many faces of interaction

11. Supporting the learner and teacher online

12. Making a mark - taking assessment to technology

13. Continuing professional development and ICT: target practice

14. Assuring quality

Section D: A connected future

15. Nine years of DentEd: a global perspective

16. A vision of dental education in the third millenium
}

PART 1.

\section{DIGITAL CLINICAL RECORDS}

\section{Crucial design features}

Successful clinical dental practice requires high levels of competence in both manual and cognitive* skills. However, until recently, research has led to developments in materials, equipment and techniques for the enhancement of manual rather than cognitive skills. Improved instruments and better designed dental units are two examples. It has now been realised that information and communication technology (ICT) can enhance clinicians' cognitive skills, such as those required during diagnosis, treatment planning and treatment.

\section{${ }^{*}$ Cognition can be defined as 'the process of knowing in the broadest sense, including perception, memory} judgment, etc.'

Professor, Faculty of Dentistry, University of British Columbia, Vancouver, Canada and Dental School, Greifswald University, Greifswald, Germany; ${ }^{2}$ Honorary Senior Research Fellow, School of Dental Sciences, University of Liverpool, Pembroke Place, Liverpool, L3 5PS; ${ }^{3{ }^{*} V i s i t i n g ~ P r o f e s s o r, ~ U C L ~ E a s t m a n ~ D e n t a l ~ I n s t i t u t e, ~}$ 256 Gray's Inn Road, London, WC1X 8LD

${ }^{*}$ Correspondence to: Professor K. A. Eaton Email: K.Eaton@eastman.ucl.ac.uk

\section{Refereed Paper}

DOI: $10.1038 /$ sj.bdj.2008.243

${ }^{\circledR}$ British Dental Journal 2008; 204: 387-395
In order to identify how to support the cognitive processes that a dental clinician uses during diagnosis, decisionmaking, treatment planning and clinical management, these processes have to be analysed and their strengths and weaknesses identified. It is then possible to design systems to support and enhance clinical cognitive performance and thus improve the quality of care.

Unfortunately, until now, many of the ICT-based applications have lacked integration because they have not been based on sound physiologic-ergonomic as well as cognitive-ergonomic principles. In other words, they have not been designed in an integrated manner that enables dental clinicians to work and think with maximum ease. Poor design has resulted in the creation of ICT-based 'island solutions' in which the physical components, such as intra-oral cameras, digital radiographic systems and clinical records, do not combine into an ergonomically and cognitively integrated system.

Against this background, the first part of this paper presents a concept of chairside communication that harnesses sound physiologic-ergonomic and cognitive-ergonomic principles which integrate all the ICT-based components. 
The core of this integrated system is a complete clinical dental record system, comprising medical and dental history, diagnosis, treatment planning, progress notes and patient maintenance. This ICTbased clinical documentation provides a comprehensive set of retrospective evaluation procedures, quality assessment and development without additional work away from the chairside. More than $90 \%$ of the content is common to dental practice in all countries. The digital clinical record can therefore easily be transferred from one country or language to another. It also links to any digital administrative system for billing or management. The system can be integrated in dental units and is run either by voice-recognition and feedback or in wireless mouse mode.

\section{Human cognitive performance}

Holistic understanding and decisionmaking are important parts of human cognitive competence. Human cognitive processes can be described as taking place on two levels, an upper, conscious level and a lower, subconscious level.

At the upper level, processes such as inferencing and decision-making can be performed. However, this capacity is very limited both with regard to information processing and to shortterm memory, where only six to eight information chunks can be kept in mind simultaneously. In addition, processes are executed sequentially. ${ }^{1}$ A typical example of a process at the conscious level is deciding whether or not tooth 14 (upper first pre-molar) should be extracted in a given clinical situation.

The lower subconscious level is characterised by almost unlimited capacity. Here an immense number of information processes are taking place simultaneously. They are controlled by the upper level through a complex, autopilot-like monitoring and control system. If something inadequate occurs during the performance of a process at the lower level, which the autopilot cannot handle, the upper level is interrupted and starts appropriate corrective actions. A typical example of this process is using the handpiece when preparing a cavity. If a patient's head movement is only slight, adaptation by the clinician takes place automatically under the control of the 'autopilot'. If the head movement is too large or sudden, an interrupt is issued and the conscious level is activated for appropriate intervention.

Clearly, optimum working conditions require that in any situation, information is presented in such a way that it can be understood and managed by processes at the lower level. In addition, minimum use must be made of the upper (conscious) level, in order to hold this capacity free to deal with any unexpected problems. If working conditions comprise the performance of the upper level (calculating and making inferences and decisions), they should not overload the limited capacity available.

In most cases, clinical managementrelated decision-making implies simultaneous handling of far more than eight information chunks. This implies that dental clinicians cannot generate a holistic view and understanding of a patient's clinical situation from a conventional paper-based or electronic patient record. Clinical data and information must therefore be transformed to sensory and, in particular, visual representations. ${ }^{2-4}$

\section{Supporting clinical cognitive performance}

In order to identify the need for support of cognitive activities, one of the authors of this paper (I-VW) has analysed clinical activity and problems with regard to the strengths and weaknesses of human cognitive performance. The results have clearly revealed that the paper-based patient record severely hampers diagnosis and decision-making with regard to the choice and execution of appropriate clinical management. ${ }^{5}$

For effective integrated treatment planning, oral clinicians need to adopt a holistic understanding of the patient's complaints and expectations, their medical, dental and social histories, risk factors, the soft tissues of their mouth and their occlusion as well as the coronal, apical, periodontal, and radiographic status of their teeth. These factors represent far more than the six to eight 'information chunks' that can be handled simultaneously by the upper level of cognitive processes in the short-term memory. It is therefore easy to see why paper-based records, in most cases, inhibit the process.

Holistic comprehension of at least the coronal, apical, periodontal and radiographic status of teeth can only be achieved by representing clinical findings with appropriately coloured graphic symbols, and by presenting these symbols simultaneously in such a way that the information can be grasped and managed by processes at the lower (subconscious) level. Intuitively this has been known for a long time. Methods for graphically documenting oral status on paper-based records have therefore been used in most practices. However, paper and pencil impose severe limitations. A transition to electronic records is, therefore, clearly indicated.

Unfortunately, in reality, most of the computerised medical and dental records that have been developed have worsened the situation. This is due to the fact that conventional methods of information analysis and alphanumeric computer technology have been applied with no real consideration to the needs of the dentist now and in the future. It almost seems as if current computerised dental records and charts were produced with the sole objective of automating conventional paper systems. This objective renders the electronic record very 'familiar' to the practitioner but it fails to take the additional capabilities of ICT into account.

As has been explained, a digital clinical record should aim to enhance a clinician's cognitive performance. As such it builds upon people's intuitive abilities. Such a digital clinical record system, based on recent research into humancomputer interaction, ${ }^{3,4,6-8}$ has been developed and will now be described.

\section{The 'computerised dental practice'}

In general, a 'computerised dental practice' has included a series of diagnostic instruments, intra-oral cameras, digital radiographic systems, treatment planning systems, CAD-CAM systems, management systems, etc. These represent 'island solutions', which have not been integrated into one system and have not been planned to support the cognitive process. 
All these island solutions are characterised by the lack of physiologicergonomic and cognitive-ergonomic principles. Typically, they have included intra-oral cameras on mobile stations with trailing cables that have to be moved to the dental units, together with screens with clinical information that can only be seen by the clinician if he or she moves his or her head and keyboards that present potential cross infection control problems and disturb the clinical routine. All these are clear examples of a lack of physiologic-ergonomic concepts.

Even worse, these island solutions generate time consuming computer dialogs that have to be answered in order to display one, two, but unfortunately never more than six radiographs on the screen. Until now digital radiographic systems have not saved time in the daily practice due to the lack of cognitive-ergonomic concepts. ${ }^{9}$ In other words, computer programs for clinical dental practice must be physiologic-ergonomically integrated into the clinical environment. In addition, they must be designed to accommodate the clinical workflow as well as based on cognitive-ergonomic principles.

In order to address this unsatisfactory situation, a team from Uppsala University and a German publishing company (Quintessenz Verlags-GmbH, Berlin, Germany) developed the concept of 'All in one - Chair-side Communication' based on established physiologicergonomic, cognitive-ergonomic and workflow-based principles. The system was successfully tested in 12 clinical environments in eight European countries in the European Research and Development Project ORQUEST (19961998). ${ }^{9-13}$ It is based on a standardised ICT-platform and provides immediate availability of all clinical information required for diagnosis, treatment and recall at the chairside. As mentioned previously, the system is built on physiologic as well as cognitiveergonomic, workflow-based principles, eg using an ergonomically optimally integrated flat screen, as well as a voicebased human-computer dialogue. ${ }^{9-12}$ It has subsequently been marketed by a Finnish dental company (Planmeca, Helsinki, Finland).

\section{Features of chairside communication based on physiologic-ergonomic and cognitive-ergonomic principles}

This section of the paper considers how 'peripherals' should be positioned around a dental chair to ensure their optimal physiologic-ergometric and congnitive-ergometric use. Generally, when treating patients, dental clinicians sit in the so-called 9-, 10- or 11-o'clock positions. If they have a screen to look at, it has to be in a 3-or 4-o'clock position. This position enables access to clinical information without turning the head; one has just to raise the eyes from the patient's mouth to the screen. For patient education the screen can easily be moved to the 6-o'clock position so that both clinician and patient can view the screen together. A handle allows the screen to be turned to face the dental nurse (chairside assistant), when necessary.

In the near future data entry by dictation (voice recognition) will be a reality. A small 'earphone' will inform the clinician, via a human voice simulation, what the computer has understood. Corrections during dictation are easily made without interrupting the oral examination. In spite of the fact that voice recognition is a very comfortable method of data entry, a mouse- or digitiser-based data entry system must be available at the chairside (eg for image manipulation). For mouse- or digitiser-based data entry, a data entry procedure is required which allows the dental nurse to keep pace with the clinician's dictation. This can be accomplished by special cognitive-ergonomically designed data entry procedures. It must be pointed out that it is not possible to keep pace with dictation by using keyboard or conventional touch-screens. ${ }^{5}$

For mouse- or digitiser-based data entry, a small mouse tray is located on both sides of the dental chair, so that it can be easily available to both clinician and dental nurse. As with all the other peripheral ICT-based equipment, which will be described, the position of the mouse tray has been designed to be optimally integrated at the chairside both physiologically and ergonomically. The mouse tray is placed under the chair and is easy to move for data entry. The intra-oral camera, as an ICT-support for clinical documentation, diagnostics, and patient education, is immediately available in the same way as handpieces.

Intra-oral digital radiography needs to be available at the chairside and/or in a special radiographic room equipped with intra- and extra-oral digital radiographic systems.

From the cognitive-ergonomic point of view it is extremely important that all clinical information, such as the clinical dental record, radiographs, photographs etc, are immediately available and accessible at the chairside. It is well known that the basis for appropriate clinical management is a continuous holistic understanding of a patient's clinical condition. This requires that the presentation and navigation of all information in the clinical record, including risk factors, intra- and extra-oral radiographs and intra- and extra-oral images, are based on established cognitive-ergonomic, workflow-based principles in order to be immediately accessed, easily managed, and quickly grasped and understood 'in one view'.

Figure 1 shows the clinical interface at the chairside. On the upper part of the screen there is the presentation area for the clinical record comprising medical and dental history, oral status (charting), treatment plan and progress notes.

The medical and dental history includes a comprehensive summary of information that is of direct relevance to care delivered in dental practices. The selection and presentation of the elements within the medical and dental history must be facilitated in such a way that the patient's chief complaint, the reason for the visit, past dental experiences (eg frequency of visits and previous treatment), dental phobia and family history can be easily understood in any clinical workstation and considered during treatment planning. The clinician can classify a patient to be 'at risk' by marking specific items (conditions) in the history with the label 'alert'. This triggers an automatic display of these conditions by the system at the top of the screen whenever clinical information concerning the patient is presented. 
The documentation of oral status in the form of graphic symbols is a wellknown method that has been used in most conventional practices. The use of different colours and easily-understood symbols facilitates a systematic understanding of the patient's mouth. It has also shown that a holistic presentation of coronal, periodontal, endodontic and radiographic status in one single graphic promotes integrated clinical thinking and simplifies treatment planning.

The graphical presentation of the oral status used in digital clinical records must aim to enhance a clinician's information processing performance and build upon their intuitive perceptual ability. An appropriately designed graphical presentation of a patient's mouth must enable the dentist to recognise and understand the patient's clinical situation in one view with very low cognitive strain.

Treatment planning can then be carried out, based on the risk factors, the oral status graphic and the image material (radiographs and photographs) made available by the system. Special treatment planning menus, which set out possible alternative plans and list the clinical procedures that they involve, are available.

An important and extensive part of the patient record is found in the progress notes section of the system. The progress notes consist of all clinical treatment procedures to be updated. They are represented by a hierarchy of sets of cognitively related terms (Fig. 2). Each set forms a menu. When new progress notes are added they automatically update the oral status graphic (eg if an amalgam restoration is documented by selecting the treatment steps from the progress notes menu, caries will disappear from the oral status graphic and a graphic of the amalgam restoration will appear). Additionally, there is an automatic link to update patient billing via open interface links to any billing or management system.

As previously mentioned, more than $90 \%$ of the content of the progress notes is common to all countries. The digital clinical record can therefore easily be transferred from one country or language to another.

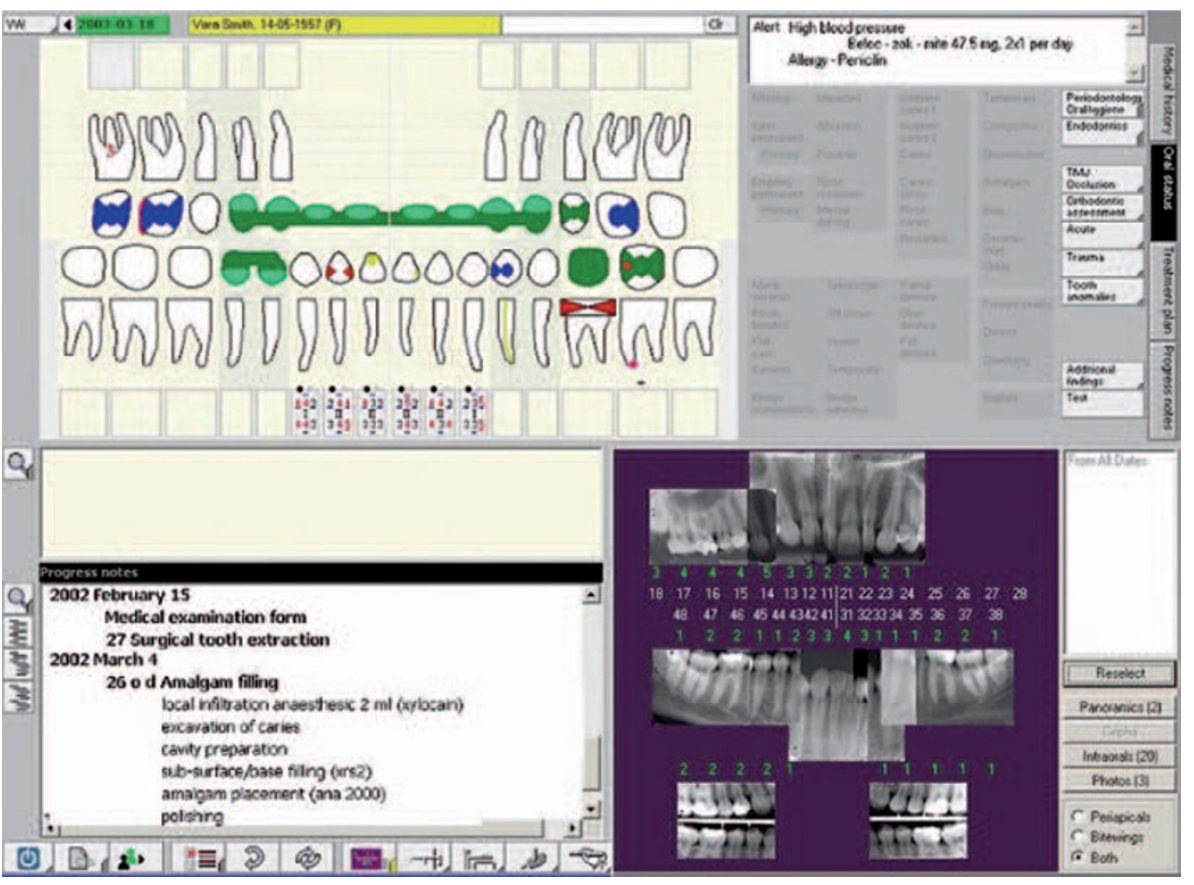

Fig. 1 Clinical interface at the chairside

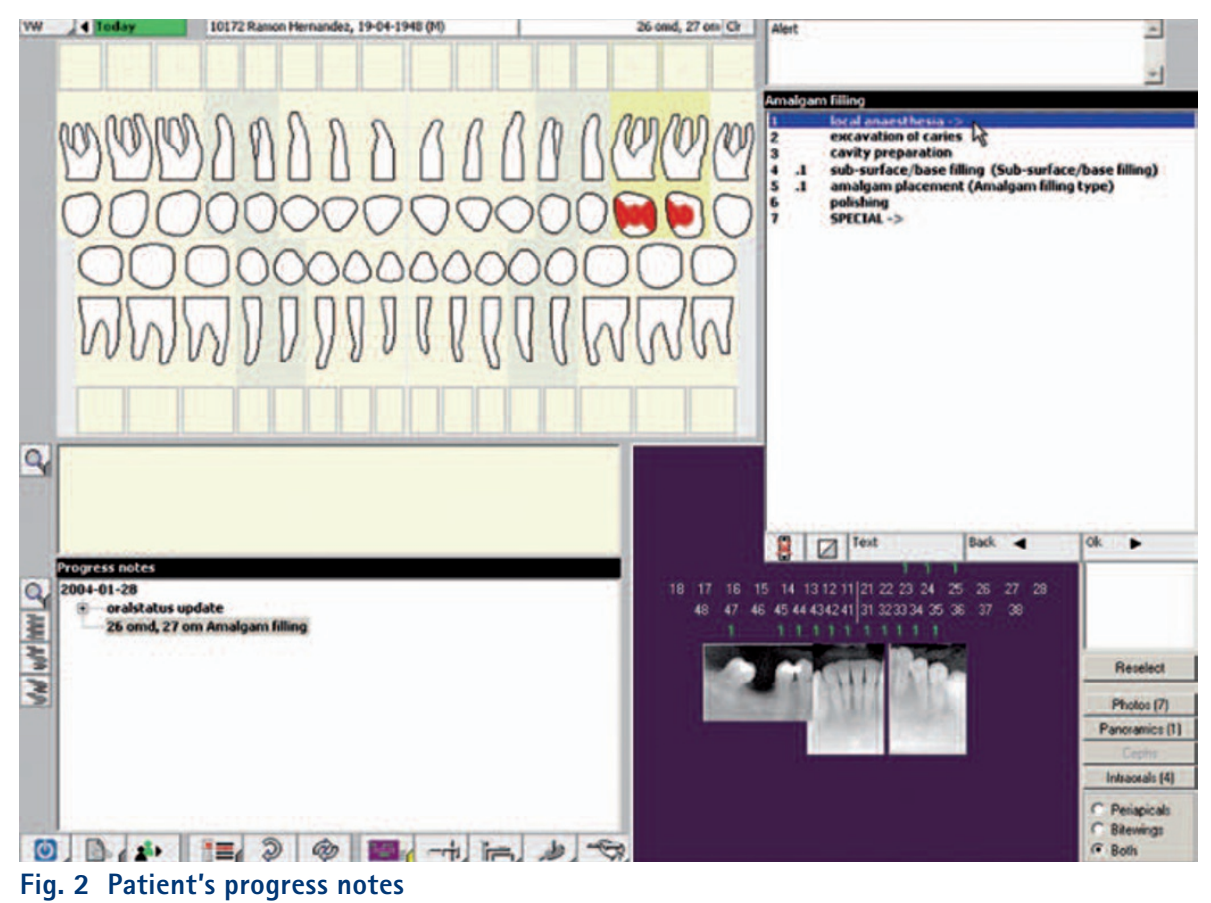

In the lower right region of the screen (Fig. 1) the patient's intra-oral radiographs (periapicals and bite-wings) are displayed in the form of an 'intuitive image-archive'. This presentation gives a synoptic overview of the teeth/ regions for which there are radiographs. A green number indicates that there are more radiographs available for a specific region. For regions where no radiographs exist, the space is left empty. For full mouth sets of radiographs, a panel indicates the dates when the radiographs were taken. For diagnostic interpretation and comparison the images can be magnified. By clicking on the dates or the tooth regions of interest, the corresponding radiographs are displayed immediately. The user has the possibility of displaying several radiographs from the same set (horizontal overview), or selecting images of one tooth region at different dates and comparing them (vertical overview). The patient's panoramic radiographs, cephalographs and photographs are also immediately accessible. Should a clinician need help with regard to the interpretation of a 
radiograph, an image and rule-based radiographic decision support system for the clinical management of radiographic lesions ${ }^{14}$ can be linked to the digital clinical record.

The use of an intra-oral camera, other imaging systems, diagnosis and patient education must be physiologic-ergonomically and optimally integrated at the chairside. The following situation demonstrates this need: a dentist takes an intra-oral image and detects an oral mucosal lesion but does not feel confident with regard to diagnosis and clinical management. At this moment an image- and rule-based decision support system for the diagnosis and treatment of oral mucosal lesions ${ }^{15,16}$ should be immediately accessible and linked to the actual intra-oral image. This decision support system works as follows: The clinician is asked two questions about location and colour of the lesion in question. After answering, the computer selects those diseases/lesions which are characterised by these symptoms and presents them simultaneously on the screen. On the screen the dentist now compares the image of the actual clinical lesion with the clinical images selected by the support system. He/she then selects the image which best matches the clinical lesion. Now the program displays a number of images describing different forms and stages of the selected disease/ lesion. In addition, the most important facts about the condition and information concerning clinical management are shown together with the possible differential diagnosis. It should be emphasised that it is not the aim of this decision support system to make general practitioners specialists on oral mucosal lesions. Quite the contrary, the aim is to make him/her secure with regard to the further clinical management of the patient by access to specific data and information which often only belongs within the domain of the specialist.

Last but not least, digital clinical records, including medical/dental histories, oral status findings, treatment planning and progress notes, are a prerequisite for quality assessment and development and facilitate both clinical audit and research. These procedures are far easier if the records have been produced by a system in which the design is based on established physiologic- and cognitive-ergonomic concepts.

A clinical record system based on the cognitive-ergonomic principles outlined above is at present under implementation at the Dental School of British Columbia University (UBC) in Canada.

\section{PART 2. DIGITAL PRACTICE MANAGEMENT AND ADMINISTRATION}

\section{Introduction}

Historically in the UK and most other countries, digital clinical records have been established as a development of administrative systems, which in turn have been initiated as a result of demands or incentives from external funding authorities such as the National Health Service (NHS) in the UK and Medicaid in the USA. This has resulted in the 'island solutions' referred to in the first part of this paper. Over the last 15 years, the uptake of computerised practice administration systems in general dental practice has been rapid. In 1991, in England and Wales, 23\% of dental practices had computers and this had risen to $60 \%$ in $1998 .{ }^{17}$ It is now almost certain that nearly $100 \%$ of dental practices rely on computers to support their administrative systems.

The establishment of integrated digital clinical records affords an efficient and ergonomic solution to providing administrative data without duplication which can be extracted for use internally in the clinic or practice. This usually takes place outside the immediate clinical environment and externally to the clinic but can link the clinic to advisory or supporting bodies. Effective and efficient oral health care can only be provided in the primary care setting if the administrative aspects of the practice or clinic are given appropriate attention. This can only be achieved effectively if the benefits of ICT solutions are seen as part of a fully integrated system. This not only eliminates the possibility of the potential duplication of information, with the consequent reduction in efficiency, but also allows the transfer of information within the administrative system, both internally within the practice or clinic environment and also externally, for example to funding authorities, financial advisors and monitors of educational activity.

Although digital practice administration should be viewed as an integrated system, it is easiest to consider the many applications individually and how these might impact on the ergonomics and efficiency of practice administration and the impact on improving the quality of healthcare.

\section{Financial management}

\section{Financial management}

for the patient

It has become increasingly important that patients are not only informed about the cost of treatment that has been undertaken by means of an efficient and accurate billing system, but also that they are fully informed about the proposed treatment and the costs involved, prior to agreeing to receive it. A comprehensive estimate of the treatment, which might include a number of different treatment options, can take considerable time to prepare if reliance is placed on a paper-based system. This also has the added disadvantage of possibly requiring a duplication of the time taken, due to the necessity for the dentist to retain a copy of the estimate for future reference. An ICT solution can easily translate the results of a chair-side discussion by delivering a written estimate to the practice administrative area without the expenditure of additional time by either the clinician or the administrative staff. In addition, a digital system can record that an estimate has been printed and given to the patient. This can effectively eliminate confusion and misunderstanding, which so often can lead to a patient/ clinician breakdown in communication.

\section{Financial management for the practice}

The digital patient record can be linked to a billing system providing immediate information for the practice, the patient and, where appropriate, for the funding authority. The development of relational spreadsheets by linking to financial software packages allows fast and accurate tracking of income and expenditure. This addresses the issues of 
efficient cash flow, sophisticated financial planning and forecasting and can significantly reduce accountancy and external financial advisors' costs.

\section{Office management}

Links with the electronic patient record provide the opportunity to print out personalised prescriptions, reports and letters using templates and stored patient data, together with increased and more efficient access to patient information throughout the practice. Informative practice information leaflets and newsletters, providing useful and up-to-date patient information, can be printed on demand using word processing or publishing software packages.

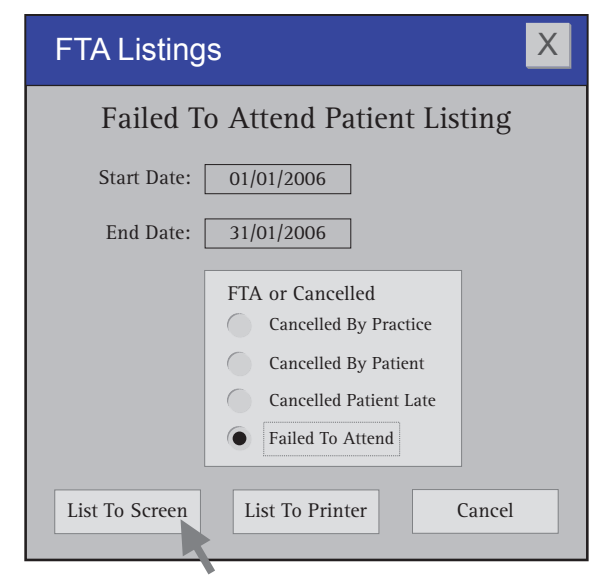

Fig. 3 Schematic example of the failed to attend patient listing

\section{Appointments and patient appointment information}

Appointments can be requested by means of a practice website and a digital appointment system can be established which conforms to the needs of both the patient and the clinician using efficient and accurate search facilities. Patients can be notified of appointments and automated reminders implemented using email or automated telephone reminders where appointments are made a long time in advance or where a significant amount of time has been reserved. This can result in a more efficient utilisation of clinical time.

\section{Stock control}

ICT applications provide efficient systems to monitor appropriate stock levels and stock rotation combined with direct electronic ordering links to chosen suppliers. If all stock usage is recorded in a stock control database, a re-order printout can be easily and quickly generated or the stock requirements can be communicated by directly interlinking the clinic and suppliers databases.

\section{Practice websites}

These can provide patient information in the home or business environment including: the booking of appointments, out of hours and general health care

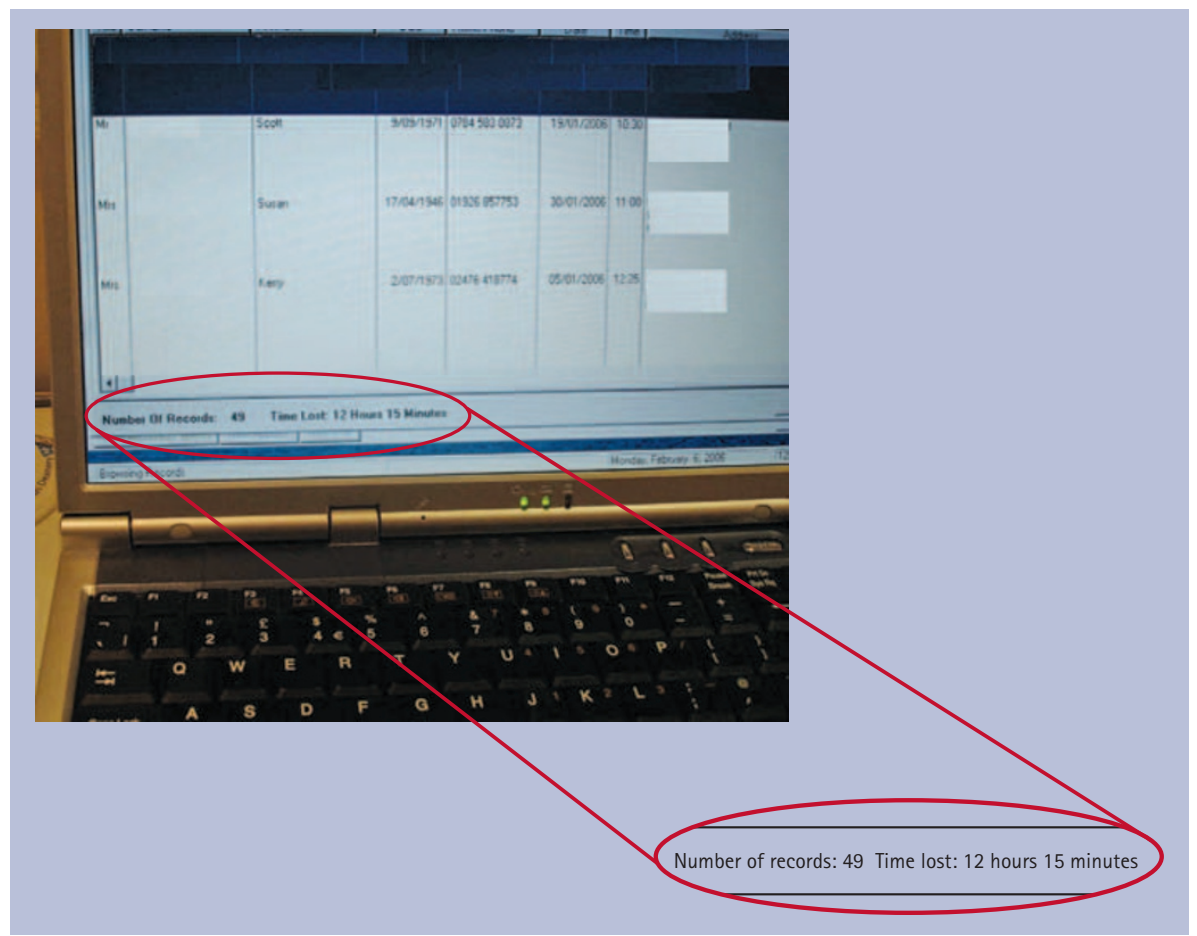

Fig. 4 Outcome of audit on patients failing to attend advice, practice information, policy, etc. Such a website can have the added effect of enhancing the practice image and providing opportunities for online patient oral health education.

\section{Continuing professional development (CPD)}

ICT can provide an avenue for all members of the dental team to obtain verifiable and non-verifiable CPD and to provide a log which can be produced as evidence of all such activity. Examples of the provision of ICT-based CPD are discussed in depth in other papers in this series.

\section{Clinical governance}

Clinical governance is defined by the Department of Health as 'A framework through which National Health Service (NHS) organisations are accountable for continuously improving the quality of their services and safeguarding high standards by creating an environment in which excellence in clinical care will flourish'. ${ }^{18}$ This definition is not confined to care provided within the NHS but can be extended to apply to treatment provided under any payment system. In trying to implement continuous quality improvement it is necessary to establish minimal standards for both clinical and administrative care which are nationally or locally accepted.

The advantage of utilising ICT solutions is that the defined and accepted standards can be incorporated into the ICT system such that the system is programmed to prevent the clinician or any member of the dental team from accepting data input which falls short of the prescribed standards. For example, many hand-written clinical patient records are deficient on important registration data such as telephone numbers, post codes etc. An electronic data entry form can be programmed to prevent the user from progressing until this important data is registered correctly. To further improve the quality of the data recording, the dental record can benefit from being made interactive, ie the administrator can be prompted to record all the appropriate data. This removes the requirement of having numerous check lists which might be necessary when using 
paper-based systems. This is important if the data is going to be used for retrospective analysis since an incomplete database will result in a flawed data analysis. In addition, the updating of the local or nationally accepted standards can be undertaken both quickly and efficiently so that the quality of care is continuously improved.

\section{Audit}

Audit, whether clinical or administrative, is an essential part of the continuous improvement of quality of care. Unfortunately the collection of data using a paper-based system can be very time consuming and therefore expensive. This can provide a powerful disincentive to undertake the exercise as frequently or extensively as might be desirable. The utilisation of ICT solutions in the form of practice or clinic administration software can enable historical data to be obtained without any additional input of time by the administration staff. For example, an audit could be undertaken to establish the number of failed patient appointments over a specific period of time. This can be requested from the computerised patient database by only a few key strokes (Fig. 3) and the result produced immediately, not only indicating the total number of failed appointments but also the amount of clinical time lost and therefore the economic impact on the clinic (Fig. 4). To undertake this procedure manually, without the benefit of a computerised database, could take considerable administrative time and thereby reduce the potential gain of undertaking the analysis.

When an audit is undertaken, for example, looking at the quality of clinical radiographs, the results will probably show that the majority of the radiographs are clinically satisfactory, some will probably be very good and a small minority could be of little or no diagnostic value (Fig. 5). When the results are analysed and changes made to rectify the deficiencies identified, a second audit can be carried out. This should show an overall quality improvement with the best results in the first audit being exceeded by the best results in the second audit (Fig. 6). This graphically demonstrates a quality improvement

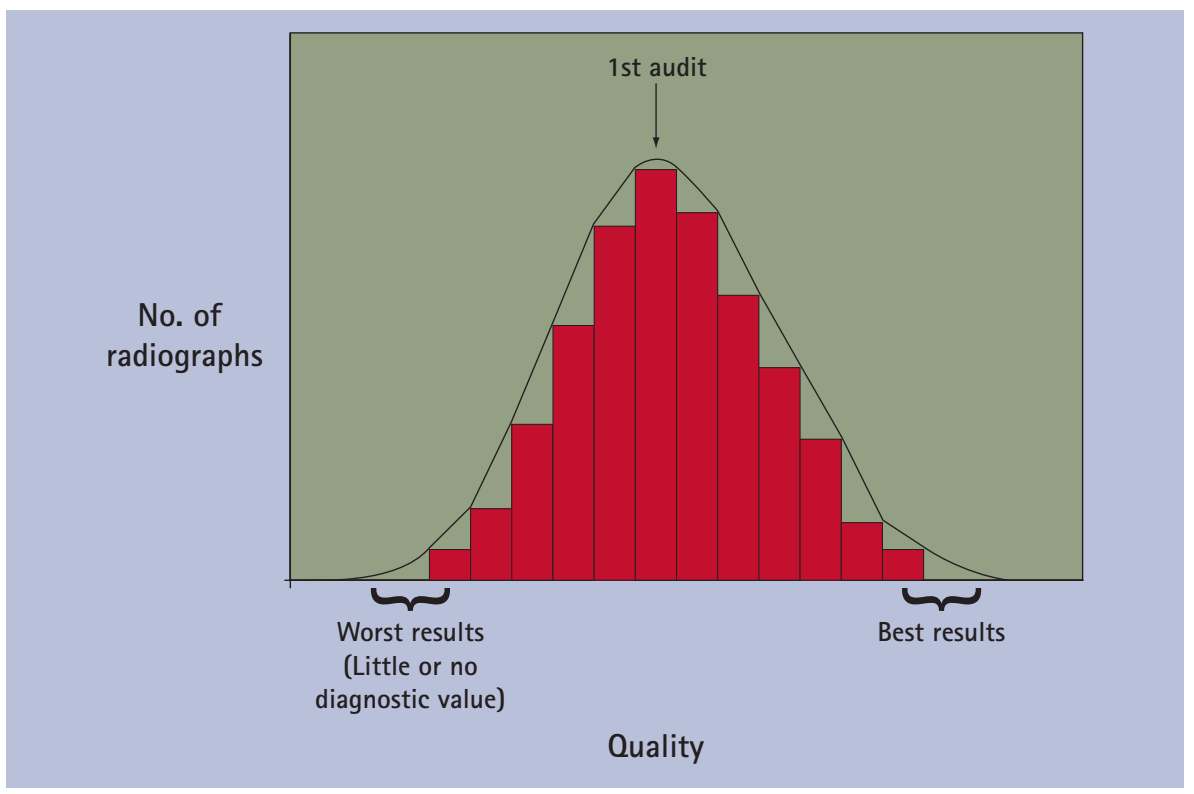

Fig. 5 Quality development through audit

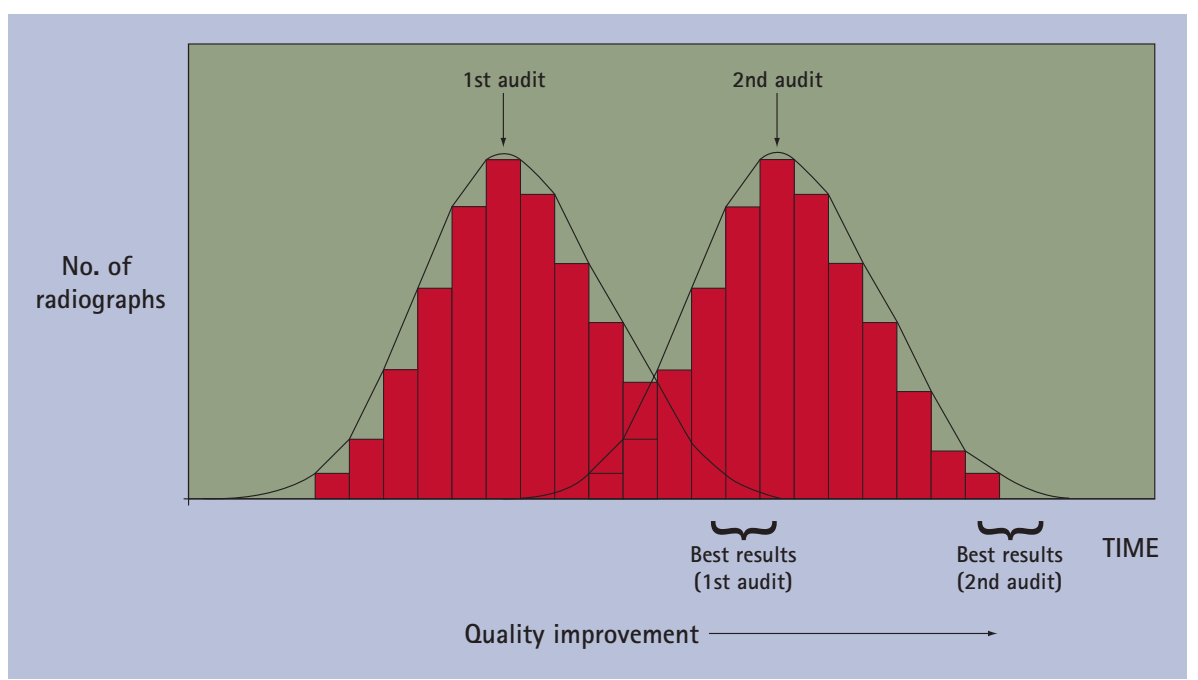

Fig. 6 Quality development through audit

with time and the results can be judged against professionally agreed standards.

ICT has the potential to offer opportunities for quality standards of both administrative and clinical care to be compared between different primary care providers working in a similar environment. To undertake this, it is first necessary to define and achieve consensus for quality indicators. These can then be recorded as part of the routine clinical data recording during the examination and treatment of the patient. A clinical minimum data set for primary dental care in the UK was defined in $2001^{19}$ and clinical quality indicators have been used as a means of recording a patient's oral health status in routine clinical practice. ${ }^{20}$ The comparison between different providers has been demonstrated to be feasible as part of the Euro-Qual BIO-MED II project, ${ }^{21}$ which looked specifically at quality indicators in orthodontics. One of the quality indicators was the incidence of white spot lesions encountered as a result of the presence of fixed orthodontic appliances. In the pilot study, a number of providers in several European countries participated by recording the incidence of white spot lesions over a defined period of time as part of routine clinical examination using a computerised clinical database. The results were then electronically transmitted to a central database, collated, compared and anonymised to reveal a considerable difference in white spot incidence reported by individual clinicians (Fig. 7). Each clinician was informed of his or her code 


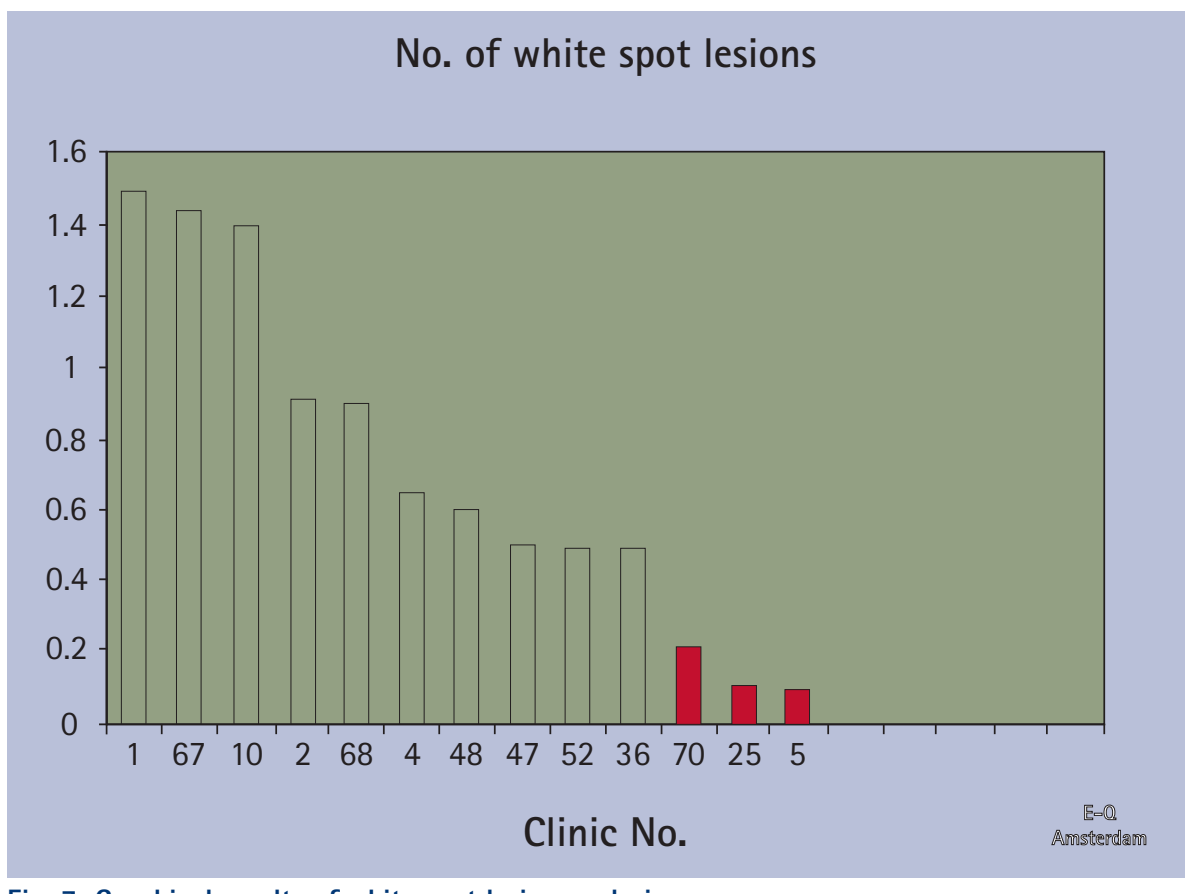

Fig. 7 Graphical results of white spot lesion analysis

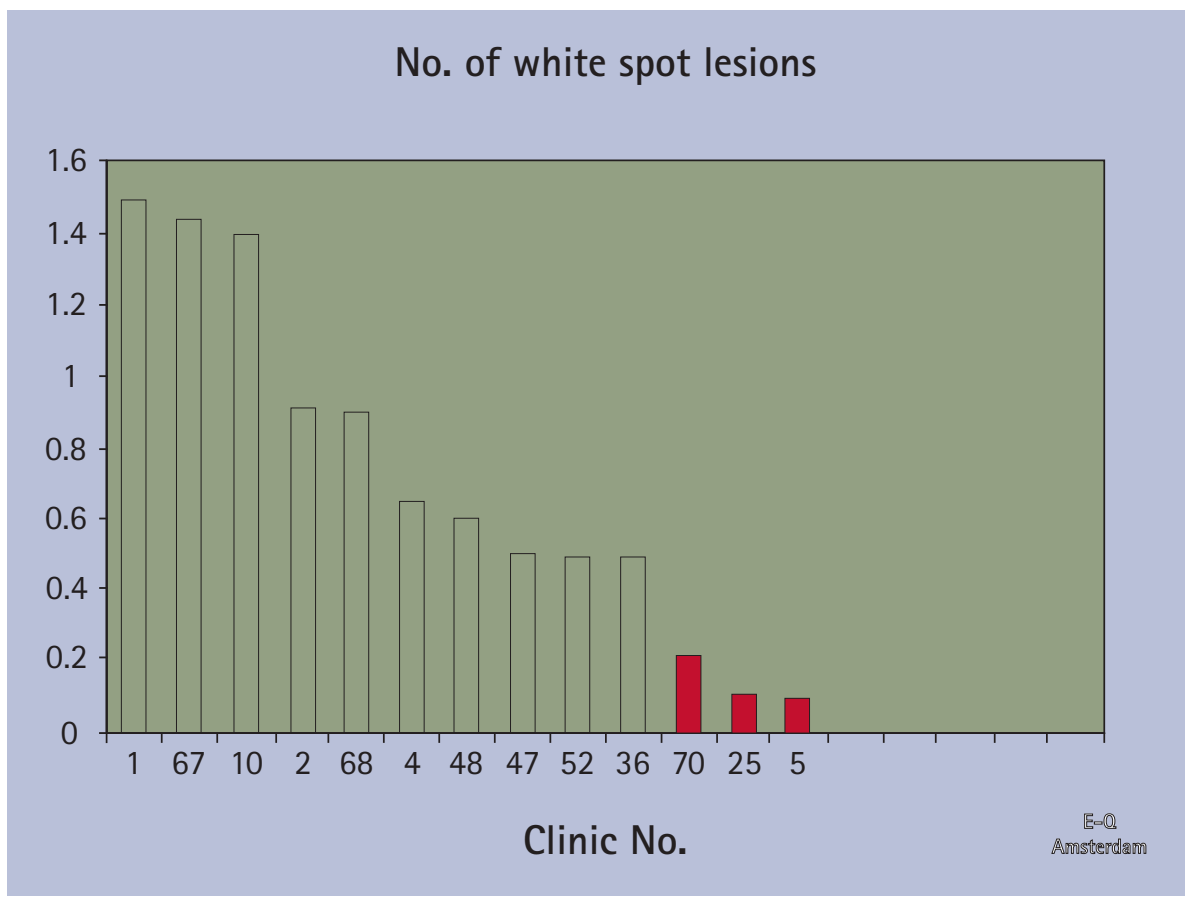

Fig. 8 White spot analysis with clinic 1 highlighted

number and was able to see their comparative results from the graph generated: for example, clinic 1 highlighted in blue (Fig. 8), performed least well of all clinics participating in the study. This clearly demonstrated the disparity with other clinicians which would otherwise have not been apparent. In the pursuit of quality improvement, and subject to their consent, it would be possible for the poorer performers, such as clinicians 1 and 67 to contact the best performers, clinicians 25 and 5, and establish systems would facilitate the formation of networks of healthcare providers capable of aggregating clinical databases. These providers would be committed to recording outcomes and thereby establishing best practice with the aim of improving health gain. ${ }^{22}$

\section{The potential disadvantages of IT solutions}

The decision to be dependent upon ICT solutions for practice administration must take into account the potential disadvantages of such a development. These include:

- The initial expense of installing appropriate software and hardware and the ongoing costs of updating and maintenance

- The lack of versatility in the software package in meeting the specific needs of an individual operator or practice

- The need for frequent electronic backup to avoid potential data loss

- The need for additional staff training, the problems of staff adaptation to change and the problems of adjusting to, understanding and managing new and complex technology

- The fragility in the software market possibly resulting in a need to change to a new system and the potential problems of compatibility in transferring data onto a new software package

- The difficulty in transferring patient data between different software systems when patients join or leave a practice

- The pressure on the software developers to adapt to changes in the requirements of third party payers.

Although clinical data transfer between different software systems still remains a challenge, the problem of transfer of administrative data has largely been solved. The other disadvantages largely involve the inevitable transition to an electronic environment and can be addressed by attention to the management of technological change inherent in a dynamic profession subject to such constant change as dentistry.

\section{SUMMARY}

In this paper, the authors have demonstrated the importance of innovative, 
adequate and appropriate use of ICT in dental practice, both with regard to clinical records and practice management and administration. One key feature for success is a holistic strategy providing for integration of various, existing as well as future, applications. Another key feature is that all applications are based on increasingly well-established knowledge with regard to human cognitive ergonomics. There are some potential disadvantages, which by no means should be disregarded. However, these can be dealt with in an appropriate way, and should not detract from the significant advantages that arise from the use of digital clinical records as well as digital practice management and administration.

The authors wish to thank Quintessence Publishing Co. Ltd. for permission to reproduce Figures 1 and 2 and sections of the narrative on pages 388, 389 and 390 of this paper which appeared in a paper by the first author published in the International Journal of Computerized Dentistry in $2006 .^{6}$

1. Miller $G$ A. The magic number seven, plus or minus two: some limits on our capacity for processing information. Psychol Rev 1956; 63: 81-97.

2. Schneider W, Wagner I-V. The use of electronic clinical recording and pro-active learning in dentistry and dental education. In Scott J J (ed). Proceedings of the 19th Meeting of the Association for Dental Education in Europe, 23-25.9.1993, Reims. pp 29-35. Ireland: Atrium Productions
Ltd./University of Dublin, Trinity College, 1993.

3. Wagner I-V, Schneider W. New concepts of multimedia- and telematics-based medical worksations. In Lemke H U, Vannier M W, Inamura K, Farman A $\mathrm{G}$ (eds) Proceedings of CAR'97 - Computer Assisted Radiology. pp 317-322. Amerstam: Elsevier Science B.V., 1997.

4. Nygren E. From paper to computer screen. Human information processing and user interface design In Acta University Uppsala: Comprehensive Summaries of Uppsala Dissertations from the Faculty of Science and Technology. Uppsala: University of Uppsala, 1996. ISBN 91-554-3699-4.

5. Wagner I-V. Internal report. Work analysis in clinical dental practice. Medical Academy of Dresden, Germany, 1989.

6. Wagner I-V. Quality assurance and quality enhancement by use of digital clinical documentation. Int J Comput Dent 2006; 9: 195-204.

7. Lind M, Petterson E, Sandblad B, Schneider W. Computer based workstations in health care. Holland: Elsevier Science Publishers B.V. (North Holland), IFIP-IMIA, 1988.

8. Wagner I-V. The use of information technology for continuous improvement of patient care. In Abbey L M, Zimmerman J L (eds) Dental informatics. pp 77-91. New York: Springer-Verlag, 1992.

9. European Research and Development Project ORQUEST (1996-1998). European Commission - DG XIII Telematic Applications Programme, Health Care - project number 1037.

10. Wagner I-V, Schneider W, Balconi D, Han F, Koch S. ALL in ONE - das Europäische Projekt ORQUEST. Computer in Zahnarzpraxis und Dentallabor - EDV-Jahrbuch, 98. pp 80-83. Germany: Hüthig Verlag, 1998.

11. Wagner I-V, Schneider W, Ammann A. Der zahnärztliche Arbeitsplatz der Zukunft: Chairside Kommunikation. In Herrlinger, Koschnitzke, Schmidt, Schwarz (eds) Praxis Ambiente. pp 323-325. Berlin: Quintessenz Verlag, 1999.

12. Koch S, Wagner I-V, Schneider W, Han F. New concept of an integrated IT\&T-based dental workstation for quality assurance in oral health care. In Cesnik B, McGray A T, Scherrer J-R (eds) Proceedings of the 9th World Congress on Medical Informatics, Seoul, Korea, August 18-22 1998. pp 107-111.

13. Wagner I-V, Schneider W, Koch S, Balconi D, Han F. Der Einsatz multimedialer Informationstechnologie für Diagnostik und Therapie zur Qualitätsentwicklung im klinischen Management - das europäische Projekt ORQUEST. ZMK (Magazin für Zahnheilkunde, Management und Kultur) 1998; 6: 34-42.

14. White $S$, Wagner I-V, van der Stelt P. Decision support for interpretation and clinical management of radiographic lesions. (CD-ROM) Berlin: Quintessenz Verlags-GmbH, 2003.

15. Wagner I-V, Schneider W. Computer-based decision support in dentistry. In Preston J (ed) Proceedings of the First International Conference on Computers in Clinical Dentistry. Chicago: Quintessence Publishing Co., 1993.

16. Straßburg M, Wagner I-V, Schneider W. Mundschleimhauterkrankungen - Entscheidungsunterstützung für die tägliche Praxis. Das große Lehrbuch interaktiv auf $C D-R O M$. Berlin: Quintessenz Verlags-GmbH, 1997.

17. Dental Practice Board. Dental computer survey. London: Department of Health NHS Management Executive, 1998.

18. Department of Health. Providing assurance on clinical governance: a practical guide for internal auditors. London: Department of Health, 2005. Electronic version only. Gateway ref. 4721.

19. Ireland R S, Jenner A M, Williams M J, Tickle M. A clinical minimum data set for primary dental care. BrDent J 2001; 190: 663-667.

20. Burke F J, Busby M, McHugh S, Delargy S, Mullins $A$, Matthews R. Evaluation of an oral health scoring system by dentists in general dental practice. Br Dent J 2003: 194: 215-218.

21. Njio B J, Stenvik A, Ireland R S, Prahl-Andersen B (eds). Euro-Qual. European orthodontic quality manual. IOS press, 1999. ISBN 9051994788.

22. Peterson P E. Continuous improvement of oral health care in Europe. J Int Dent Assoc 1994; 40: 105-107. 